\title{
Three-dimensional distinct element simulation of spherocylinder crystallization
}

Received: 6 May 2004 / Published online: 17 February 2005

(C) Springer-Verlag 2005

\begin{abstract}
We present a three-dimensional distinct element model (DEM) able to handle populations of spherocylinders. We report on granular crystallization occurring when vibrating mono-disperse assemblies of spherocylinders that faithfully reproduce the corresponding results of physical experiments from the literature.
\end{abstract}

Keywords DEM · Granular media · Rods · Spherocyliners · Crystallization

\section{Introduction}

Granular media are highly pervasive in nature and play an important role in technology. Because of their particular behavior, they have been said to constitute a fourth state of matter. In the past, one was confined to carry out experimental studies with real grains in order to gain some insight into their behavior. With the availability of increased computational power, it has become possible to design and implement simulation models of growing realism. Although granular media present in nature are composed of grains that are all but spherical, so far most simulation models were only able to handle spherical grains. This is a large limitation, since many interesting phenomena cannot be captured by such models. In this paper we present a new three-dimensional distinct element simulation method that can handle non-spherical grains and is able to produce simulation experiments that confirm findings reported using real such granular media. This demonstrates that it is possible to simulate large-scale heterogeneous populations composed of spheres and spherocylinders of various size and shape parameters. In particular, we correctly reproduce the rearrangement of populations of spherocylinders

L. Pournin (四) $\cdot$ M. Weber $\cdot$ M. Tsukahara $\cdot$ M. Ramaioli

Th. M. Liebling

EPFL - Ecole Polytechnique Fédérale de Lausanne,

Mathematics Institute, $\mathrm{CH}-1015$ Lausanne, Switzerland

E-mail: lionel.pournin@epfl.ch

J.-A. Ferrez

IDIAP Research Institute, Martigny, Switzerland subject to tapping. This paper is organized as follows: first, we give a quick review of existing literature, then we describe our simulation model and experiments we carried out with it and finally we discuss the corresponding results.

\section{Literature review}

Probably the first distinct element simulation model can be found in [2]. The main contribution of that model was its way to handle contacts experienced by particles. It consists in considering pairs of spheres in contact as constituted of linear springs and dashpots generating forces both normal and tangential to the contact and similarly for the moments. This approach will be extended to contacts between spherocylinders and described in more detail in section 3. In [10] and later in [3] this model was implemented using power diagrams for particle contact detection, thereby yielding a particularly efficient code. The contact force models were discussed in[13], where the authors point at problems with the dissipative behavior of the modeled media and propose a way to alleviate them. Recently and independently of us, [1] proposed a Metropolis-Monte-Carlo method to simulate the steady-state distribution of populations of spherocylindrical grains. This approach makes no use of any explicit mechanical properties other than particle weight and is purely geometric in what regards contacts. Some of the steady state distributions they report turn out to correspond to metastable states. In contrast, physical experiments were carried out [20] wherein a stable distribution is obtained which happens to be crystalline. The authors of [1] did not find this distribution because reaching it with their Monte-Carlo approach would certainly have taken prohibitively long simulation times. Indeed leaving a metastable state to reach a stable one requires going through many highly improbable transitions in their model.

\section{Simulation method}

We propose here an extension of the distinct element method able to handle spherocylinder assemblies. A spherocylinder 


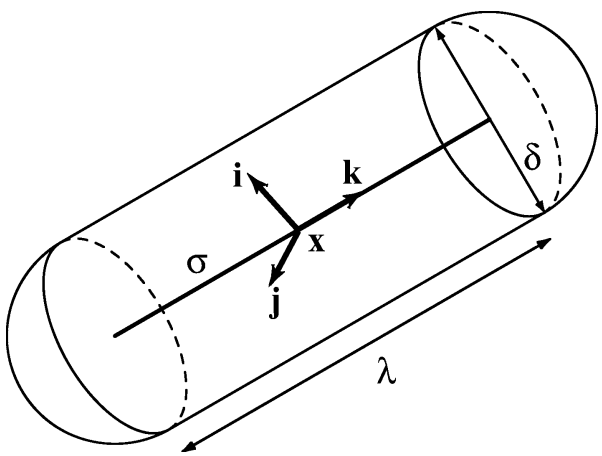

Fig. 1 A spherocylinder of characteristic diameter $\delta$ and length $\lambda$ and the referential $(\boldsymbol{x}, \boldsymbol{i}, \boldsymbol{j}, \boldsymbol{k})$

is defined as follows. Let $\sigma$ be a line segment in $\mathbb{R}^{3}$ and $\delta$ a positive real. The spherocylinder of shaft $\sigma$ and diameter $\delta$ (radius $\delta / 2$ ) is the set of points whose distance to $\sigma$ is smaller or equal to $\delta / 2$ (Figure 1). The length of $\sigma$ is called $\lambda$ as on figure 1. In other words a spherocylinder is the Minkowski sum of the line segment $\sigma$ with a centered ball of diameter $\delta$.

The distinct element method iteratively updates the state of the medium, whose geometrical constituents are grains and walls. At every time-step, the contacts between the elements are detected and values for the resulting contact forces are calculated. The model assumes those forces remain constant for the duration $\Delta t$ of the time-step. The evolution of the state of the medium is then computed by numerical integration of Newton's laws of motion for each individual grain. The process is repeated at the next step with this new configuration. A rough flow chart of the simulation process is given in Figure 2.

A main issue encountered when simulating granular media is the choice of a suited physical contact model. In DEM, grains are assumed to be homogeneous, deformable solids. A grain which is in contact with others or with a wall experiences deformation. In general, precise determination

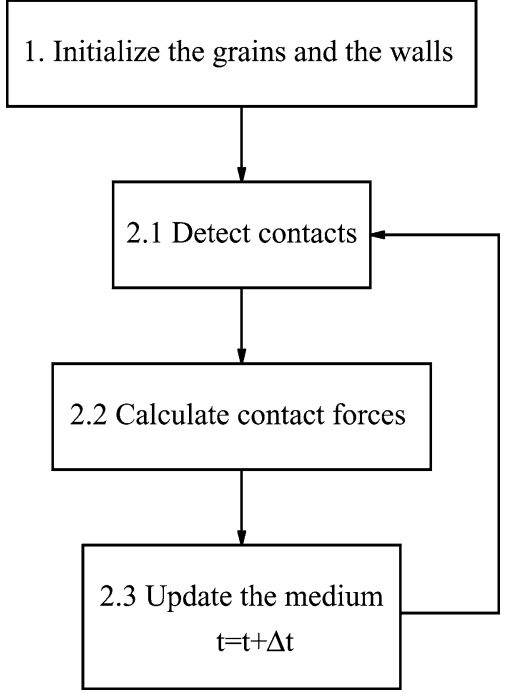

Fig. 2 Simulation algorithm of the contact deformation is not trivial. However, as stated in [2], the behavior of a granular medium as a whole is more due to the motions of the particles than to their deformations which are small by comparison. Hence, precise knowledge of deformation is not necessary for a good approximation of mechanical behavior of the medium. In the model, a contact will occur whenever two objects (two grains or a grain and a wall) overlap. This overlap is taken as a measure of the deformation of the particles at the contact point (see figure 3). The contact force is then modeled by a force-overlap relation, taking into account the overlap and its time derivative. A motivation to model grains with spherocylinders is that an overlap can easily be defined with this particular shape. Two spheres overlap when the distance between the grain centers is smaller than the sum of their radii. Analogously, two spherocylinders will overlap when the distance between their shafts is smaller than the sum of their radii.

As shown in Figure 2, at every iteration of a simulation, one must detect all the contacts between pairs of grains. One first possibility is to check for contacts between each pair of grains. This leads to contact detection in $O\left(n^{2}\right)$ time where $n$ is the number of grains. The complexity of detection can be reduced by considering subdivisions of the simulation space so that grains that are in contact may only be found in adjacent cells. However, one must track the grains that are in each cell. Quadtrees are successive subdivisions of the simulation space until there is no more than one grain per cell. A very efficient algorithm for detecting contacts uses dynamic triangulations so that grains which are in contact are connected by an edge $[10,4]$.

Implementation of the model was based on the program previously developed by the authors [4] for the simulation of spherical grains. Work on a triangulation-based contact detection for spherocylinders is in progress. However, a spatial sorting algorithm was preferred to obtain the results reported in section 5. This method consists in enclosing each spherocylinder in a ball and testing those pairs of spherocylinders for contacts whose enclosing balls intersect. In order to find the pairs of enclosing balls which intersect, we chose a naive method which has the virtue of being very simple. While not adequate for large grain populations, this method is quite suited for our purposes since it allows to treat cases with 1000 to 2000 grains without much overhead. Taking some direction $\boldsymbol{d}$ as the altitude, it consists in sorting the balls in decreasing order with respect to their highest point. They are then scanned in this order and a given ball $b$ is only tested for contact with those balls whose highest point lies above the lowest point of $b$. As the balls are sorted, we obtain a mean complexity of $O\left(n^{\frac{5}{3}}\right)$, which is nearly quadratic but reveals helpful in practical cases.

\subsection{Physical contact model}

Consider two spherocylindrical grains $G_{i}, i=1,2$ (given by their characteristic diameter $\delta_{i}$, half-shafts $\boldsymbol{a}_{i}$, position $\boldsymbol{x}_{i}$ of the mass center, shaft $\sigma_{i}$, linear velocity $\boldsymbol{v}_{i}$ and spin vector $\boldsymbol{\omega}_{i}$ ) experiencing a contact (Figure 3). 


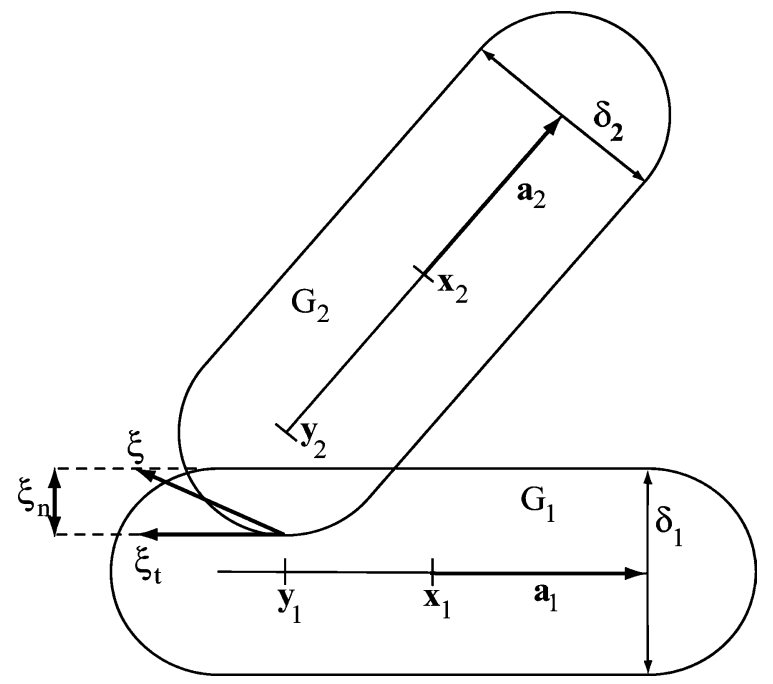

Fig. 3 A contact between two spherocylinders

Let $\boldsymbol{f}_{G_{1} \rightarrow G_{2}}$ and $\boldsymbol{f}_{G_{2} \rightarrow G_{1}}$ be the forces applied by $G_{1}$ on $G_{2}$ and by $G_{2}$ on $G_{1}$ respectively. Newton's third law gives $\boldsymbol{f}_{G_{1} \rightarrow G_{2}}=-\boldsymbol{f}_{G_{2} \rightarrow G_{1}}$, and we denote $\boldsymbol{f}=\boldsymbol{f}_{G_{1} \rightarrow G_{2}}$. In order to model those contact forces and give their application points on either grain, we need to define some parameters which will quantify the geometry of the contact area. In particular, we need to find two points $\boldsymbol{y}_{1} \in \sigma_{1}$ and $\boldsymbol{y}_{2} \in \sigma_{2}$ for which the shortest distance between $\sigma_{1}$ and $\sigma_{2}$ is attained, that is, $\left\|\boldsymbol{y}_{1}-\boldsymbol{y}_{2}\right\|=d\left(\sigma_{1}, \sigma_{2}\right)$.

Let us first consider the case when the shafts $\sigma_{1}$ and $\sigma_{2}$ of $G_{1}$ and $G_{2}$ are in general position. In this case, the pair $\left(\boldsymbol{y}_{1}, \boldsymbol{y}_{2}\right)$ is unique. Let $\psi_{\sigma_{1}}: s \mapsto \boldsymbol{x}_{1}+s \boldsymbol{a}_{1}$ for $s \in[-1,1]$ and $\psi_{\sigma_{2}}: t \mapsto \boldsymbol{x}_{2}+t \boldsymbol{a}_{2}$ for $t \in[-1,1]$ be parametrizations of $\sigma_{1}$ and $\sigma_{2}$. We suppose that the shafts are non-degenerate, that is to say $\boldsymbol{a}_{1} \neq 0$ and $\boldsymbol{a}_{2} \neq 0$ otherwise the computation is either trivial or reduces to finding the point on a line segment closest to another point. Finding $\boldsymbol{y}_{1}$ and $\boldsymbol{y}_{2}$ amounts to minimizing $\left\|\psi_{\sigma_{1}}(s)-\psi_{\sigma_{2}}(t)\right\|^{2}$ for $(s, t) \in[-1,1]^{2}$. A solution to this optimization problem was previously derived [7,19]. Letting $s^{*}$ and $t^{*}$ be the optimal solution, $\boldsymbol{y}_{1}$ and $\boldsymbol{y}_{2}$ are given by: $\boldsymbol{y}_{1}=\psi_{\sigma_{1}}\left(s^{*}\right)$ and $\boldsymbol{y}_{2}=\psi_{\sigma_{2}}\left(t^{*}\right)$. Here is the procedure we use:

Input: $\boldsymbol{x}_{1}, \boldsymbol{a}_{1}, \boldsymbol{x}_{2}, \boldsymbol{a}_{2}$

Output: $\left(s^{*}, t^{*}\right) \in[-1,1]^{2}$ so that $\left\|\sigma_{1}\left(s^{*}\right)-\sigma_{2}\left(t^{*}\right)\right\|$ is minimal.

1. Compute $a=a_{1}^{2}, b=-a_{1} a_{2}, c=a_{2}^{2}, d=a_{1}\left(x_{1}-x_{2}\right)$, $e=-\boldsymbol{a}_{2}\left(\boldsymbol{x}_{1}-\boldsymbol{x}_{2}\right), \delta=a c-b^{2}$.

2. Compute $t^{*}=\alpha\left(\frac{b d-a e}{\delta}\right)$ and $s^{*}=\alpha\left(\frac{-b t^{*}-d}{a}\right)$. If $\frac{-b t^{*}-d}{a} \notin$ $[-1,1]$, compute $t^{*}=\alpha\left(\frac{-b s^{*}-e}{c}\right)$. Stop.

Here $\alpha$ is defined as:

$\alpha(u)= \begin{cases}-1 & \text { if } u \leq-1 \\ u & \text { if } u \in]-1,1[. \\ 1 & \text { if } u \geq 1\end{cases}$

Now suppose $\sigma_{1}$ and $\sigma_{2}$ are parallel. In this case, we may have several choices for the pair $\left(\boldsymbol{y}_{1}, \boldsymbol{y}_{2}\right)$. Let $p: \sigma_{1} \rightarrow \mathcal{D}$ be the projection of $\sigma_{1}$ on the line $\mathcal{D}$ supporting segment $\sigma_{2}$. If $p\left(\sigma_{1}\right) \cap \sigma_{2}=\emptyset$ then the $y_{2}$ is the point on $\sigma_{2}$ closest to $p\left(\sigma_{1}\right)$ and $y_{1}$ is the point on $\sigma_{1}$ whose image under $p$ is the point of $p\left(\sigma_{1}\right)$ closest to $\sigma_{2}$. If $p\left(\sigma_{1}\right) \cap \sigma_{2} \neq \emptyset$, we choose $y_{2}$ as the center of $p\left(\sigma_{1}\right) \cap \sigma_{2}$ and $y_{1}$ as the point on $\sigma_{1}$ which projects on $y_{2}$.

Knowing $y_{1}$ and $y_{2}$, we define the unit vector "normal" to the contact as:

$\boldsymbol{u}_{n}=\frac{\boldsymbol{y}_{2}-\boldsymbol{y}_{1}}{\left\|\boldsymbol{y}_{2}-\boldsymbol{y}_{1}\right\|}$

For tangent spherocylinders, $\boldsymbol{u}_{n}$ is the normal vector to the tangent plane. We define the application points of forces $\boldsymbol{f}_{G_{1} \rightarrow G_{2}}$ and $\boldsymbol{f}_{G_{2} \rightarrow G_{1}}$ as the intersections of the segment $\left[y_{1}, y_{2}\right]$ with the boundaries of $G_{2}$ and $G_{1}$, that is respectively $\boldsymbol{c}_{2}=\boldsymbol{y}_{2}-r_{2} \boldsymbol{u}_{n}$ and $\boldsymbol{c}_{1}=\boldsymbol{y}_{1}+r_{1} \boldsymbol{u}_{n}$. The relative velocity $\boldsymbol{v}_{r}$ at the contact is introduced as the velocity of $\boldsymbol{c}_{2}$ with respect to $c_{1}$,

$$
\begin{aligned}
\boldsymbol{v}_{r}= & \boldsymbol{v}_{2}-\boldsymbol{v}_{1}-\left(\boldsymbol{y}_{2}-\boldsymbol{x}_{2}-r_{2} \boldsymbol{u}_{n}\right) \wedge \boldsymbol{\omega}_{2} \\
& +\left(\boldsymbol{y}_{1}-\boldsymbol{x}_{1}+r_{1} \boldsymbol{u}_{n}\right) \wedge \boldsymbol{\omega}_{1}
\end{aligned}
$$

The overlap $\xi$ is defined as the solution of the differential equation

$\dot{\xi}=-\boldsymbol{v}_{r}$

for which $\xi=\mathbf{0}$ when the contact begins. The normal and tangential overlaps are the projections of $\boldsymbol{\xi}$ on $\boldsymbol{u}_{n}$ and on the plane perpendicular to $\boldsymbol{u}_{n}, \xi_{n}=\boldsymbol{\xi} \cdot \boldsymbol{u}_{n}$ and $\boldsymbol{\xi}_{t}=\boldsymbol{\xi}-\left(\boldsymbol{\xi} \cdot \boldsymbol{u}_{n}\right) \boldsymbol{u}_{n}$. We assume that $\boldsymbol{u}_{n}$ is constant during a contact. Then the time derivatives of the normal and tangential overlaps are

$\dot{\xi}_{n}=-\boldsymbol{v}_{r} \cdot \boldsymbol{u}_{n}$

$\dot{\boldsymbol{\xi}}_{t}=-\boldsymbol{v}_{r}+\left(\boldsymbol{v}_{r} \cdot \boldsymbol{u}_{n}\right) \boldsymbol{u}_{n}$.

Equations (2), (3) and (5) yield

$\xi_{n}=r_{1}+r_{2}-\left\|\boldsymbol{y}_{2}-\boldsymbol{y}_{1}\right\|$

which expresses the normal overlap as the length of the segment joining $c_{1}$ and $c_{2}$. Practically, throughout a contact, the time derivatives of both overlaps and the normal overlap are calculated by direct application of equations (5), (6) and (7). On the other hand, the tangential overlap is computed by numerical integration of (6).

The components of $\boldsymbol{f}$ parallel and perpendicular to $\boldsymbol{u}_{n}$, the normal force $f_{n}$ and the tangential force $f_{t}$, are calculated by a force-overlap relation. In the program, the force-overlap relation used is the linear viscoelastic model first proposed in [2], $\boldsymbol{f}_{n}=\left(k_{n} \xi_{n}+c_{n} \dot{\xi}_{n}\right) \boldsymbol{u}_{n}$ and $\boldsymbol{f}_{t}=k_{t} \boldsymbol{\xi}_{t}+c_{t} \dot{\boldsymbol{\xi}}_{t}$. We take friction into account by replacing $\boldsymbol{f}_{t}$ by $\boldsymbol{f}_{t}=\mu \frac{\left\|\boldsymbol{f}_{n}\right\|}{\left\|\boldsymbol{f}_{t}\right\|} \boldsymbol{f}_{t}$ whenever $\left\|\boldsymbol{f}_{t}\right\|>\mu\left\|\boldsymbol{f}_{n}\right\|$, where $\mu$ is the Coulomb coefficient. More details on that kind of force models, and especially about the way $k_{n}$ and $c_{n}$ may be chosen is available in [13]. For the sake of simplicity, this model takes no account of the relative orientation of the contacting grains. While the validity of this approximation should certainly be further analysed, the simulation results we obtain speak in its favour. 


\subsection{Contact between a grain and a wall}

Two types of walls are considered: planar and cylindrical walls. In the simulations performed, the shafts of the grains stay in one half-space defined by each plane and in the cylindrical space defined by each cylindrical wall. Under these conditions, a grain $G$ is in contact with a wall if and only if the distance from one or both ends of its shaft to the wall is smaller or equal to the radius of the grain. Suppose this takes place for an end $\boldsymbol{e}$. Let $d$ be the normal to the wall and passing through $e$. We define the application points of the forces acting on the grain and the wall as the intersections of $d$ with the boundary of the grain and with the wall. The contact force is calculated similarly to the contact between two grains. When overlaps exist at both ends, for example if the grain is parallel to the wall, the contact is modeled by two forces.

\subsection{Updating the state of the medium}

For each contact, applying the physical contact model yields expressions for the forces that act on the grains and the walls and their application points. Let $G$ be a grain like that of figure 1 , with mass $m$, inertia tensor $I$ and subject to a set of forces $\boldsymbol{f}_{1}, \ldots, \boldsymbol{f}_{n}$. Suppose that force $\boldsymbol{f}_{i}$ has application point $c_{i}$. The laws of motion for $G$ are

$m \ddot{\boldsymbol{x}}=m \boldsymbol{g}+\boldsymbol{f}_{1}+\cdots+\boldsymbol{f}_{n}$

$I \dot{\omega}=\left(c_{1}-x\right) \wedge f_{1}+\cdots+\left(c_{n}-x\right) \wedge f_{n}$

In the model, the trajectories of the particles are computed individually by numerical integration of equations (8) and (9). In the program, the forward Euler method is used for these integrations. The orientation of the particles is represented using quaternions.

For spherocylindrical grains, the inertia tensor expressed in the referential $(\boldsymbol{x}, \boldsymbol{i}, \boldsymbol{j}, \boldsymbol{k})$ of figure 1 is a diagonal matrix, whose diagonal elements $I_{i i}, I_{j j}$ and $I_{k k}$ are given by

$$
\left\{\begin{array}{l}
I_{i i}=\frac{1}{48} \pi \rho \delta^{2} \lambda^{3}+\frac{3}{64} \pi \rho \delta^{4} \lambda+\frac{1}{60} \pi \rho \delta^{5}+\frac{1}{24} \pi \rho \delta^{3} \lambda^{2} \\
I_{j j}=I_{i i} \\
I_{k k}=\frac{1}{32} \pi \rho \delta^{4} \lambda+\frac{1}{60} \pi \rho \delta^{5}
\end{array}\right.
$$

\section{Experiments}

We simulate the rearrangement of mono-disperse populations of spherocylinders enclosed within a cylindrical container subject to vertical tapping. In order to do this, we use a cylindrical container of base diameter $D$, which is sufficiently high so that the particles cannot escape from it. Its base is mobile and can be subject to a $30 \mathrm{~Hz}$ sinusoidal vertical oscillation whose amplitude $a$ can be chosen, whereas its vertical wall remains motionless. The grains used for the rearrangement are spherocylinders of diameter $\delta$ and elongation parameter $\phi=\lambda / \delta$. Population size is $N_{p}$. Simulation processes are initialized by randomly placing the particles within the cylinder, the directions of their shafts being uniformly distributed, and letting them settle under the action of gravity. Once the kinetic energy has dropped to zero, we start the vibrations. We monitor the intensity of the vibrations via the ratio $\Gamma=\frac{a(2 \pi \nu)^{2}}{g}$, that is the maximal acceleration brought by the bottom plane to the granular packing. Individual grains have density $\rho$ and we further define the medium's volumetric density $\rho_{m}$ as the ratio of the total volume occupied by the grains divided by the volume of the enclosing truncated cylinder (upon elimination of outliers). In order to capture the reordering behavior as a function of the height of the enclosing cylinder, the latter is subdivided into four horizontal layers of equal thickness $h=4 \mathrm{~mm}$, numbered in increasing order from bottom to top. In other words, layer $i$ comes to lie between heights $(i-1) h$ and $i h$.

We monitor the behavior of the medium in terms of the mean angle between the grain shafts and the bottom plane as well as its volumetric density $\rho_{m}$, both globally and layer by layer. Experiments $a n_{3}, \ldots, a n_{9}$ were designed to investigate the way elongated grains rearrange, and in particular to observe possible re-orderings which would recall a phase transition. Among these seven experiments, the main one is $a n_{6}$. Experiment $a n_{9}$ involves shorter grains in order to show how the elongation parameter $\phi$ changes the rearrangement behavior. In this experiment, the number of grains is chosen so that the initial filling height is closest possible to that of $a n_{6}$. The other five experiments were carried out to investigate possible influence of the restitution coefficient $e_{n}$ and the vibration intensity $\Gamma$ on the results.

The parameter values for the various simulations we carried out are given in table 1

\section{Results and discussion}

Lateral snapshots of experiment $a n_{6}$ at various evolution stages are given in figure 4. Quantitatively the spherocylinders rearrange vertically to a highly ordered state, at least along the cylinder boundary. The cuts of the cylinder with a plane through its axis shown in figure 4 further show that this particular rearrangement also occurs way to the middle of the container. Obviously the concurring shapes and sizes of both container and grains play a role on crystallization processes. In particular, this is why the spherocylinders tend to crystallize vertically, achieving a high volumetric

Table 1 Experiments of vibrating mono-disperse media

\begin{tabular}{ccccccccc}
\hline & $\Delta t(\mathrm{~s})$ & $t_{c}(\mathrm{~s})$ & $e_{n}$ & $\Gamma$ & $\phi$ & $\delta(\mathrm{m})$ & $D(\mathrm{~m})$ & $N_{p}$ \\
\hline an $_{3}$ & $2 \times 10^{-5}$ & $6 \times 10^{-4}$ & 0.1 & 2.0 & 4.00 & $0.523 \times 10^{-3}$ & $8 \times 10^{-3}$ & 1000 \\
$a_{4}$ & $2 \times 10^{-5}$ & $6 \times 10^{-4}$ & 0.2 & 2.0 & 4.00 & $0.523 \times 10^{-3}$ & $8 \times 10^{-3}$ & 1000 \\
$a_{5}$ & $2 \times 10^{-5}$ & $6 \times 10^{-4}$ & 0.4 & 1.5 & 4.00 & $0.523 \times 10^{-3}$ & $8 \times 10^{-3}$ & 1000 \\
$a_{6}$ & $2 \times 10^{-5}$ & $6 \times 10^{-4}$ & 0.4 & 2.0 & 4.00 & $0.523 \times 10^{-3}$ & $8 \times 10^{-3}$ & 1000 \\
$a_{7}$ & $2 \times 10^{-5}$ & $6 \times 10^{-4}$ & 0.4 & 2.5 & 4.00 & $0.523 \times 10^{-3}$ & $8 \times 10^{-3}$ & 1000 \\
$a_{8}$ & $2 \times 10^{-5}$ & $6 \times 10^{-4}$ & 0.8 & 2.0 & 4.00 & $0.523 \times 10^{-3}$ & $8 \times 10^{-3}$ & 1000 \\
an $_{9}$ & $2 \times 10^{-5}$ & $6 \times 10^{-4}$ & 0.4 & 2.0 & 2.83 & $0.523 \times 10^{-3}$ & $8 \times 10^{-3}$ & 1414 \\
\hline
\end{tabular}



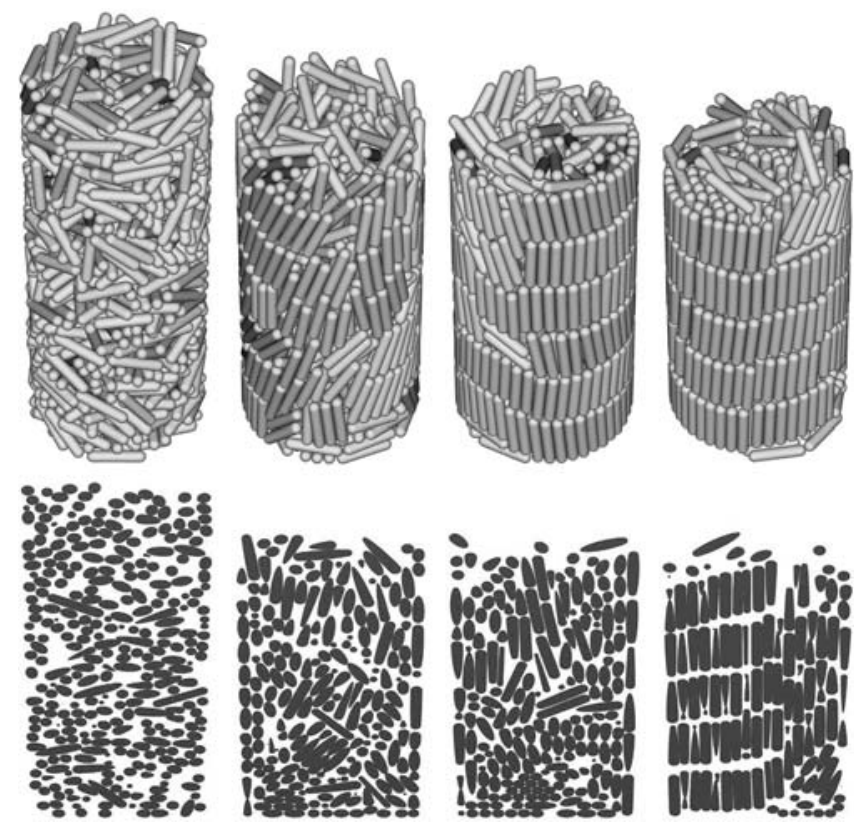

Fig. 4 Snapshots and cuts of experiment $a n_{6}$. Upper line: lateral snapshots. Lower line: corresponding cuts by a vertical plane containing the cylinder axis. From left to right: initial situation, after 300 tapping steps, after 900 tapping steps, after 4134 tapping steps
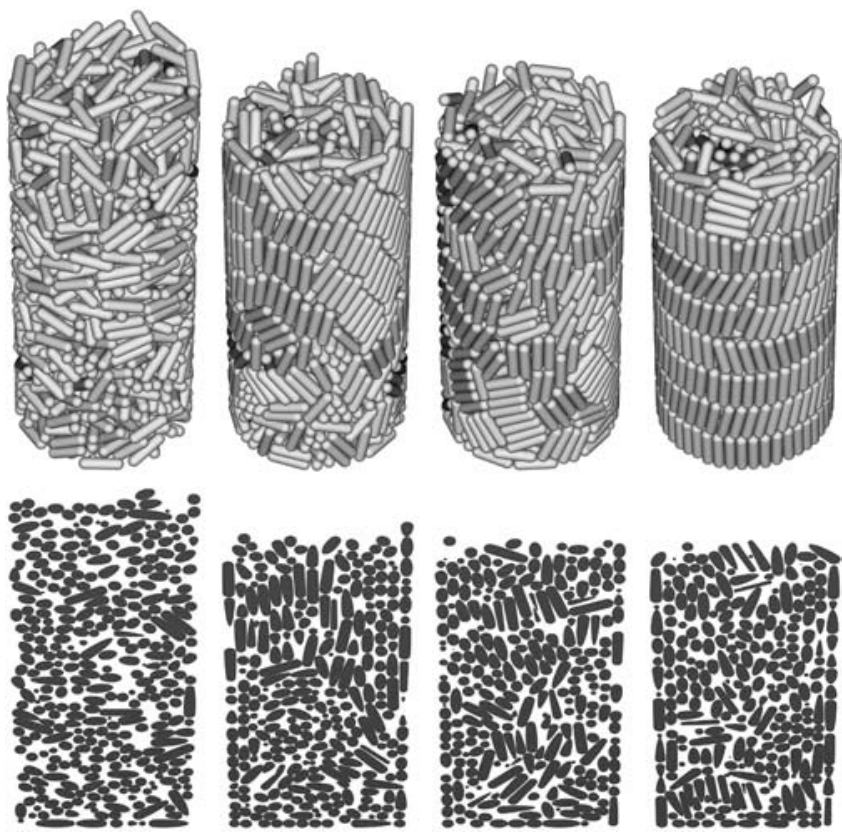

Fig. 5 Snapshots and cuts of experiment $a n_{9}$. Upper line: lateral snapshots. Lower line: corresponding cuts by a vertical plane containing the cylinder axis. From left to right: initial situation, after 300 tapping steps, after 900 tapping steps, after 4134 tapping steps

density. The final arrangements reported in [1] for Metropolis-Monte-Carlo simulations of spherocylindric populations are not crystalline. For obvious reasons one can not draw any conclusions about the dynamic behavior of real grain rearrangements from the Monte-Carlo process. Furthermore,

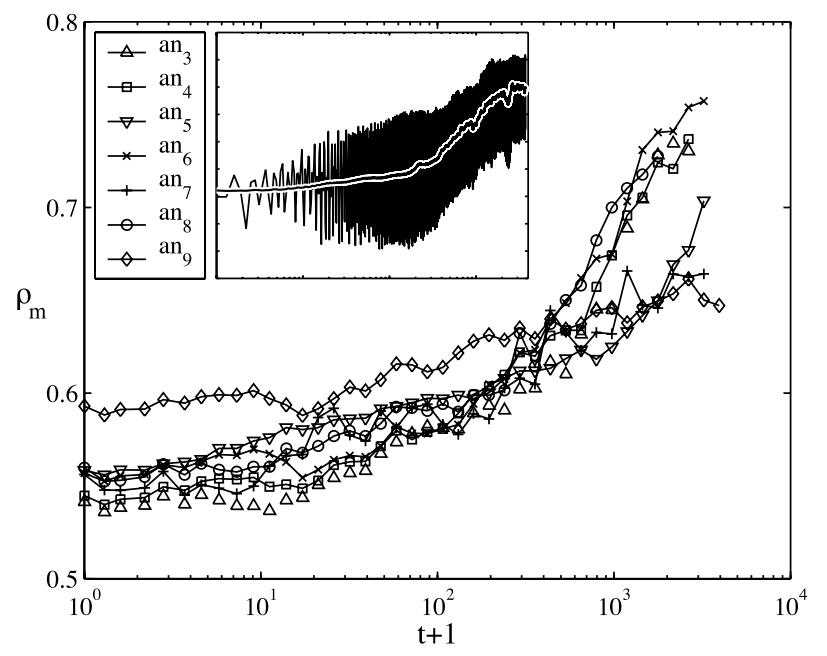

Fig. 6 Smoothed density $\rho_{m}$ plotted versus the number of tapping steps $t$ for experiments $a n_{3}-a n_{9}$. Inset: oscillations of the measured density and its moving average smoothed version for experiment $a n_{6}$

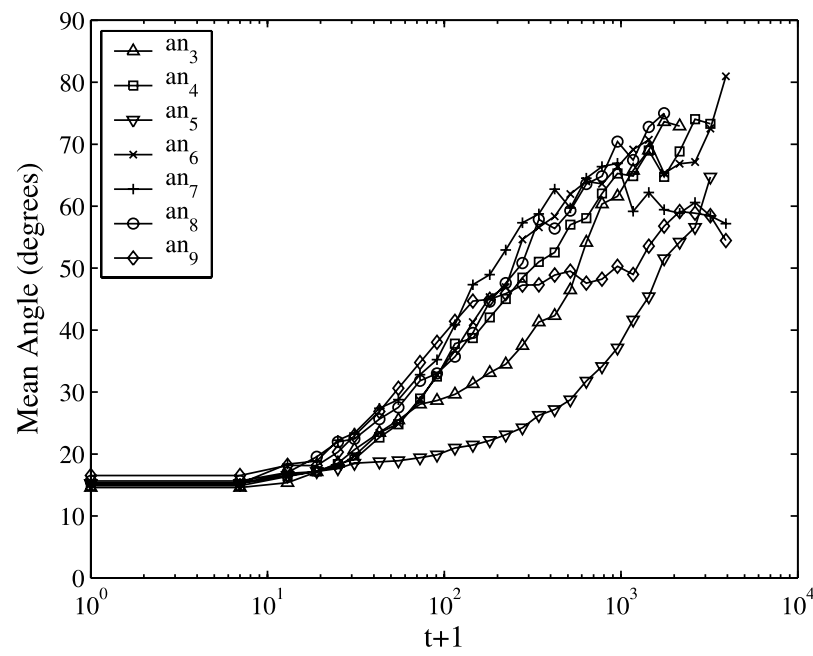

Fig. 7 Mean angle between the particles shafts and the bottom plane versus the number $t$ of tapping steps for several experiments

the final density attained in [1] corresponds to a metastable state of significantly lower volumetric density than that of the crystallized medium. This does not come as a surprise since such a metastable state is very hard to escape, the crystallization process certainly having a logarithmic behavior also in the Metropolis-Monte-Carlo simulations. The transformations in that model only allowing for rearrangements of individual grains one at a time, crystallization becomes extremely unlikely. Moreover, many of the experiments in [1] are carried out on the periodic boundary conditions, hence there are no crystalline nucleation sites, which further slows down crystallization.

We measured the volumetric density $\rho_{m}$ of the considered medium. The inset in Figure 6 shows its graph against the number of tapping steps. As can be seen, the density plot shows quite strong oscillations which can be explained by the effect of the sinusoidal tapping, the overall medium 


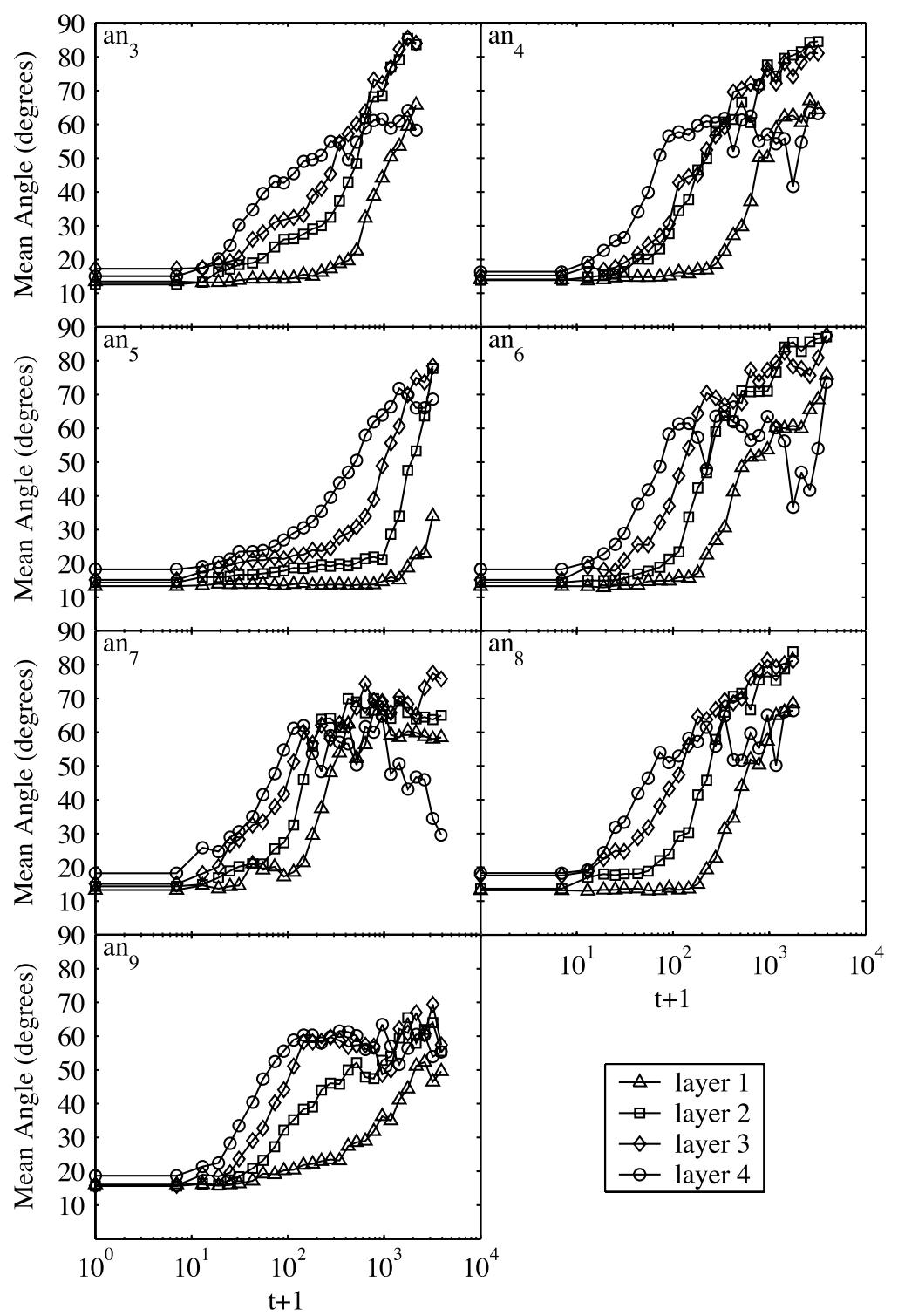

Fig. 8 Mean angle between particle shafts and the bottom plane plotted versus the number of tapping steps $t$ in four horizontal layers for experiments $a n_{3}, \ldots a n_{9}$. Layers are numbered in increasing order from bottom to top and their thickness is $h=4 \mathrm{~mm}$, that is layer $i$ is lying between heights $(i-1) . h$ and $i . h$

behaving like an elastic spring. In order to filter out this effect we smoothen the curve with a moving average as shown on the inset of Figure 6 for experiment $a n_{6}$. The smoothed version of the volumetric density $\rho_{m}$ is given in Figure 6 for the whole set of experiments.

In Figure 7 we plot the mean angle between the grain shafts and the bottom plane against the number of tapping steps. Figure 8 depicts the mean angle within several horizontal subdivisions of the container. The mean angle does not show the same oscillatory behavior as the volumetric density. A possible explanation might be that during a tapping oscillation, the angle variations tend to average out. We see that the beginning of a phase transition with respect to the angle takes place at approximately constant density (between 10 and 100 tapings). Observe that the crystallization process as measured by the volumetric density starts after about 100 tapping steps, behaving logarithmically from there on until it reaches a value as high as $\rho_{m}=0.75$ after about 2000 tapings. This value is not very far from the maximum possible density of tightly packed spherocylinders in an unbounded three-dimensional space, which equals $\rho_{m}^{\max }=\frac{\pi}{6} \frac{3 \phi+2}{\sqrt{3} \phi+\sqrt{2}}$, that is $\rho_{m}^{\max }=0.8787$ when $\phi=4.0$.

Measured by the mean angle, the crystallization process starts after 10 tapings already and behaves logarithmically until it saturates after approximately 1000 tapings for experiments $a n_{3}, a n_{5}$ and $a n_{9}$. Experiments $a n_{3}$ and $a n_{5}$ do not show a logarithmic behavior during the re-ordering process. The restitution coefficient used in experiment $a n_{3}$ is much lower than that used in the other experiments. This causes the energy reaching the grains to be small, which limits their 
motion. However, we see no dependence on $e_{n}$ for $e_{n} \geq 0.2$ (experiments $a n_{4}, a n_{6}, a n_{8}$ ). This would mean that in experiment $a n_{3}$ the energy available to the system is too small for it to reach the dynamical regime found in experiments $a n_{4}$, $a n_{6}$ and $a n_{8}$. For experiment $a n_{5}$, the value of $\Gamma$ is quite low, which means that little energy is brought to the system. In this case also, the other experiments $\left(a n_{6}, a n_{7}\right)$ show there is no dependence on $\Gamma$ for $\Gamma \geq 2.0$. Again, this suggests that the energy available to the system is not sufficient to reach the dynamics of experiments $a n_{4}, a n_{6}$ and $a n_{8}$. While experiment $a n_{9}$ exhibits the same logarithmic re-ordering as experiments $a n_{4}, a n_{6}, a n_{7}$ and $a n_{8}$, figure 7 shows that this logarithmic part is shorter and that the saturation takes place at a lower value of the mean angle. This shows that while the dynamics of the re-ordering seems not to be dependent of the elongation parameter, the saturation process is. It might be that with small values of $\Phi$, the grains have more freedom to arrange in a disordered state, and the transition to a crystalline ordering is less probable. Another possibility is that the cylinder shape has less influence on short grains than on long ones. Indeed when a slanted particle touches a cylindrical wall at two points, a torque develops that tends to erect it. The magnitude of this torque increases with particle length. It can be observed that the logarithmic stage of reordering is mainly due to global rearrangement processes (convection), whereas the saturation stage is ruled by local rearrangements. The logarithmic rearrangement behavior corresponds to that observed for media composed of identical spherical grains.

Figure 8 shows that excepting top and bottom layers, the mean angle increases monotonically to eventually stay between 80 and 90 degrees for experiments $a n_{3}, a n_{4}, a n_{6}, a n_{8}$ and between 60 and 80 degrees for experiments $a n_{5}, a n_{7}$, and $a n_{9}$. This means that the crystallization does not only occur along the container wall but involves all grains in those layers. Figure 8 also shows that in the logarithmic re-ordering stage, layers seem to reorder from the top to the bottom as the mean angle in a layer is always lower than that of the higher layers. In the saturation stage, this behavior inverts for the two central layers while the mean angle still increases in the lowest layer and drops in the topmost layer as it gradually empties. Again, this suggests that the nature of rearrangement processes in the logarithmic and saturation phases is not the same, the former being essentially due to global phenomena and the latter involving local rearrangements in a mostly static medium. This behavior matches that observed in [20] on experiments with real elongated grains. Quantitatively, the dynamics of our simulated media fits well that reported there. In particular, the time slots for both reordering processes match. The reordering is logarithmic in both cases, and the initial and final volumetric densities also agree.

\section{Conclusion}

We have presented a performing distinct element simulation scheme able to handle non-spherical grains in three dimensions, and validated this model by faithfully reproducing the crystallization of spherocylinders experimentally observed by [20]. In the setups we simulated, we observe that the crystallization process first shows a relatively fast global rearrangement stage followed by a slower phase with occasional local rearrangements. The faster phase is logarithmic whenever the energy available for the grains to move is high enough. Our simulation results raise many questions which need further investigation. They concern among others the particle trajectories during the whole process, the energy distribution within the medium, the influence of the boundary conditions, i.e. container shapes and the crystallization of grains with more complex shapes.

Acknowledgements This project was partially funded by the Swis National Science Foundation, grant \# 200020-100499. We are grateful to the referees for their very constructive remarks on an earlier version of this paper.

\section{References}

1. Abreu, C.R.A., Tavares, F.W., Castier, M.: Influence of particle shape on the packing and on the segregation of spherocylinders via Monte Carlo simulations. Powder Tech. 134, 167 (2003)

2. Cundall, P.A., Strack, O.D.L.: A discrete numerical model for granular assemblies. Géotechnique 29, 47 (1979)

3. Ferrez, J.-A.: Dynamic Triangulations For Efficient 3D Simulation Of Granular Materials. Thèse EPFL no 2432 (2001)

4. Ferrez, J.-A., Liebling, Th.M.: Dynamic triangulations for efficient collision detection among spheres with applications in granular media simulations. Phil. Mag. B 82(8), 905 (2002)

5. Lin, X., $\mathrm{Ng}$, T.-T.: A three-dimensional discrete element model using arrays of ellipsoids. Géotechnique 47(2), 319 (1997)

6. Luding, S., Clément, E., Blumen, A., Rajchenbach, J., Duran, J. Anomalous energy dissipation in molecular-dynamics simulations of grains : the "detachment" effect. Phys. Rev. E 50, 4413 (1994)

7. Lumelsky, V.J.: On fast computation of distance between line segments. Information Processing Letters 21, 1985

8. Matuttis, H.-G., Luding, S., Herrmann, H.-J.: Discrete element methods for the simulation of dense packings and heaps made of spherical and non-spherical particles. Powder Technology 109, 278 (2000)

9. Moreau, J.J.: Some numerical methods in multibody dynamics application to granular materials. Eur. J. of Mech. A-Solids 13, 93 (1994)

10. Müller, D.: Techniques informatiques efficaces pour la simulation de milieux granulaires par des méthodes d'éléments distincts. Thèse EPFL no 1545, (1996)

11. $\mathrm{Ng}, \mathrm{T}$.-T.: Numerical simulations of granular soil using elliptic particles. Computers and geotechnics 16(2), 153 (1994)

12. Ouadfel, H., Rothenburg, L.: An algorithm for detecting inter-ellipsoid contacts. Computers and geotechnics 24(4), 245 (1999)

13. Pournin, L., Liebling, Th.M., Mocellin, A.: Molecular-dynamics force models for better control of energy dissipation in numerical simulations of dense granular media. Phys. Rev. E 65, 011302 (2002)

14. Rosato, A., Prinz, F., Standburg, K.J., Swendsen, R.: Monte-Carlo simulation of particulate matter segregation. Powder Tech. 49(1) 59 (1986)

15. Rothenburg, L., Bathurst, R.J.: Numerical simulation of idealized granular assemblies with plane elliptical particles. Computers and geotechnics 11, 315 (1991)

16. Schinner, A.: Fast algorithms for the simulations of polygonal particles. Granular Matter 2, 35 (1999) 
17. Stroobants, A., Lekkerkerker, H.N.W., Frenkel, D.: Evidence for smectic order in a fluid of hard parallel spherocylinders. Phys. Rev. Let. 57(12), 1452 (1986)

18. Ting, J.M., Khwaja, M., Meachum, L.R., Rowell, J.D.: An ellipsebased discrete element model for granular materials. Int. J. for Num. and Anal. Meth. in Geomechanics 17(9), 603 (1993)
19. Vega, C., Lago, S.: A fast algorithm to evaluate the shortest distance between rods. Computers and Chemistry 18(1), 1994

20. Villarruel, F.X., Lauderdale, B.E., Mueth, D.M., Jaeger, H.M.: Compaction of rods: Relaxation and ordering in vibrated, anisotropic granular material. Phys. Rev. E 61(6), 6914 (2000) 